\title{
A Study of Coronary Heart Disease in Postmenopausal Women and Identification of Major Modifiable Determinant Risks for its Prevention
}

\author{
P Pradhan, AV Upadhya
}

\begin{abstract}
This is a retrospective study of postmenopausal women admitted with myocardial infarction (MI) in five hospitals (Nepal Medical College, Bir Hospital, Institute of Medicine, Norvic International Hospital, Medicare Hospital) having coronary care unit (CCU) in Kathmandu, Nepal, in 1 year period, from 1st Jan 2001 to 31st Dec 2001. Out of 210 cases admitted in CCU,147 (70\%) were suffering from acute MI. Two of the three following criteria were used for the diagnosis of $\mathrm{Ml}$ : characteristic substernal pain of recent origin more than 30 minutes, elevated cardiac enzymes (creatinine kinase, lactate dehydrogenase, aspirate transaminase), ECG: evidence of infarction (pathological $Q$ waves, ST elevation more than $1 \mathrm{~mm}$ in standard lead $1 / \mathrm{Avl}$ and/or ST elevation more than $2 \mathrm{~mm}$ in precordial leads V1-6 followed by $T$ wave inversion. The major identifiable modifiable risks identified are hypertension in 111 (53.8\%), smoking 73 (35.0\%), diabetes mellitus in 47 (23.03\%), hyperlipidemia in 17 (8.1\%), previous coronary heart disease (CHD) in $32(15.2 \%)$ and family history of CHD was present in $2 \%$ of the cases. Twenty-two patients (10\%) died during treatment and six patients $(3 \%)$ were readmitted with repeat MI within 6 months of the 1 st $\mathrm{Ml}$ and all recovered well.
\end{abstract}

Keywords: Myocardial infarction, Cardiovascular risks, Primary, Secondary prevention.

How to cite this article: Pradhan P, Upadhya AV. A Study of Coronary Heart Disease in Postmenopausal Women and Identification of Major Modifiable Determinant Risks for its Prevention. J South Asian Feder Menopause Soc 2013;1(2): 56-62.

\section{Source of support: Nil}

Conflict of interest: None declared

\section{INTRODUCTION}

Acute myocardial infarction (MI) remains the major cause of death in postmenopausal women in both developed and developing world. The extent and the poor prognosis of myocardial infarction in postmenopausal women have only recently been identified. Current evidence indicates that this is due to loss of protective effects of endogenous estrogen on cardiovascular system. ${ }^{1}$ Studies have shown that women are more likely than men to die after MI with both medical and surgical therapy. The in-hospital mortality rate among women who undergo coronary artery surgery is substantially higher than among men. It is uncertain whether this excess mortality reflect the older age, smaller body size, more frequent occurrence of other known coronary risk factors and perhaps the low estrogen postmenopausal state itself. ${ }^{2-8}$ Ischemic heart disease is more dependent on age in women than men, women are usually 10 years older than men. After adjusting for age, women are significantly more likely to smoke than men, women are older at enrollment, delayed presentation at hospital after the onset of symptoms and are treated 10 to 15 minutes later after arrival. These findings suggest that symptoms may be less severe in women and their significance less understood. ${ }^{1-8}$

During the past two decades there have been substantial reduction in death rates from acute MI both in men and women in USA. ${ }^{2}$ These reductions are attributable to efforts in primary prevention as well as improved therapies for MI. The importance of primary prevention is highlighted by the fact that about a quarter of new cases of coronary heart disease present as sudden death. Most primary prevention efforts should focus on the major modifiable determinants of risk of MI, e.g. cigarette smoking, hypertension, an elevated blood cholesterol level, diabetes mellitus, obesity and sedentary lifestyle. ${ }^{9}$ Attention has most recently been directed toward the possible benefit of moderate alcohol consumption and low dose aspirin prophylaxis in apparently healthy people. Secondary prevention (reducing risk in people with evidence of disease) should be approached with the same vigor as primary prevention because morbidity and mortality from MI have considerable social and financial implications for individual as well as communities. ${ }^{10,11}$

The British Cardiac Society considered that there is a considerable potential to reduce the risk of a further major coronary events in patients with established coronary disease by effective lifestyle intervention and appropriate use of prophylactic drugs. ${ }^{10-12}$ American Heart Association in 2001 reported a guideline that long-term hormone therapy is no longer recommended for either primary or secondary prevention of coronary heart disease (CHD) in women. ${ }^{13}$ This recommendation still applies based upon the result of Women Health Initiative (WHI) ${ }^{14}$ and HERS II trial ${ }^{15}$ and is consistent with the position statement of the North American Menopause Society. However, emerging data suggest that the risk of CHD events and hormone therapy are largely confined to older postmenopausal women with no significant increasing risk of CHD-related events in younger postmenopausal women. ${ }^{13-16}$ 


\section{MATERIALS AND METHODS}

All patients admitted with diagnosis of $\mathrm{MI}$ and ischemic heart disease were identified through the case notes of CCU ward in above mentioned five hospitals. Two hundred and ten women were diagnosed suffering from MI/CHD. All patients had investigations like electrocardiogram, echocardiography, cardiac enzymes, lipid profile apart from all routine radiology, blood and urine tests. MI was diagnosed if any two of the following characteristics were present; a history of substernal pain of recent onset lasting longer than 30 minutes, elevated cardiac enzymes (creatinine kinase, aspartate transaminase, lactate dehydrogenase), electrocardiographic evidence of an infarct (pathological $\mathrm{Q}$ wave and/or ST elevation of more than $1 \mathrm{~mm}$ in standard lead $1 / \mathrm{aVL}$ and/or ST elevation of more than $2 \mathrm{~mm}$ in precordial leads V1-6 followed by $\mathrm{T}$ wave inversion). Echocardiogram were done as necessary.

\section{RESULTS}

The mean age of the patient was 65 years with the range of 46 to 89 years age. The characteristics presenting symptoms were chest pain, shortness of breath, syncope, sweating and giddiness, palpitation and abdominal pain (Table 1).

Some have combination of two or three symptoms at presentations. The diagnosis was made from history, clinical examination and ECG changes and were aided by other specific investigations when needed. The diagnosis pattern is shown in Table 2.

One hundred and forty-nine (70\%) patients suffered from acute MI. The analysis of an interval between the development of symptoms and arrival to hospital varies considerably even among the patients within the city. Only $29(14.0 \%)$ patients were brought to hospital within 4 hours of developing symptoms and $14 \%$ of patient were brought

Table 1: Main presenting symptoms

\begin{tabular}{lll}
\hline Symptoms & No. & $\%$ \\
\hline Chest pain & 115 & 74.0 \\
SOB & 102 & 48.6 \\
Syncope & 28 & 33.33 \\
Sweating/giddiness & 48 & 22.4 \\
Palpitation & 14 & 6.7 \\
Abdominal pain & 4 & 1.9 \\
\hline
\end{tabular}

Table 2: Diagnosis

\begin{tabular}{lrl}
\hline Disease & No. & $\%$ \\
\hline Acute MI & 149 & 70 \\
IHD events & 26 & 12.3 \\
Angina & 22 & 10.4 \\
BBB (bilateral bundle branch) & 10 & 4.7 \\
Nonspecific & 3 & 1.4 \\
\hline Total & 210 & 100 \\
\hline
\end{tabular}

Table 3: Identifiable modifiable risks

\begin{tabular}{llc}
\hline Risks & No. & $\%$ \\
\hline Hypertension & 111 & 52.8 \\
Smoking & 73 & 34.7 \\
Diabetes & 47 & 22.3 \\
Previous IHD & 32 & 15.2 \\
Hyperlipidemia & 17 & 8.1 \\
COPD & 4 & 1.9 \\
Family history IHD & 2 & 1 \\
\hline
\end{tabular}

to hospital by 2 nd week of their illness. Identifiable modifiable risk factors analysis revealed the following risks, e.g. hypertension, smoking, diabetes mellitus, previous coronary heart disease, hyperlipidemia and obesity (Table 3).

Most common combination of risk factors were hypertension, diabetes mellitus, smoking, hyperlipidemia and obesity.

Regarding the prognosis of disease, 184 (88\%) patients recovered well. Twenty-two (10.1\%) patients died of MI during treatment. Six (3\%) patients suffered from repeat MI but recovered well.

\section{DISCUSSION}

$\mathrm{CHD}$ is an under-recognized major health problem among women in South-East Asia including Nepal. ${ }^{12}$ The prevalence of cardiovascular risk factors such as smoking, hypertension, DM, hyperlipidemia, physical inactivity and obesity has shown a significantly increased trend among women in the region. The problem is compounded by low awareness that CHD is a health problem for women as well as for men by misconception about the disease. Most primary prevention efforts should focus on the major identifiable modifiable determinant of risks of MI. ${ }^{9}$ Attention has most recently been directed toward the possible benefit of moderate alcohol consumption and low dose aspirin prophylaxis in apparently healthy people. The importance of primary prevention is highlighted by the fact that about the quarter of new cases of CHD present as sudden death. Secondary prevention should be approached with the same vigor as primary prevention because morbidity and mortality from MI have considerable social and financial implications for individual as well as communities..$^{10,11}$

\section{Indentifiable Major Determinant Risks}

\section{Smoking}

Cigarette smoking is directly responsible for $21 \%$ of all mortality from cardiac events and is inversely related to education level. Thirty-four percent of patients in this study smoked cigarette or used tobacco for more than 30 years. Data from National Health interviewed surveys indicate that 
the proportion of women starting to smoke before the age of 16 years increased from 7.2 to $20 \%$ in USA. ${ }^{17}$ In the Nurses Health Study ${ }^{6}$ it was observed that women who started smoking before the age of 15 years had the highest risk of premature death among women who were younger than 67 years. Observation case control and cohort studies have shown that smoking increases more than doubles the incidence of coronary disease and increase mortality from CHD by $70 \%{ }^{17}$ There is no clear close response relation between the number of cigarettes smoked and the risk of CHD. Smoking also acts synergistically with the other coronary risk factors, e.g. current users of oral contraceptive have about four times the risk of infarction compare to non users and women who smoke heavily and use oral contraceptive have 39 times the risk of women who do neither. The increased risk associated with smoking and diabetes, hyperlipoproteinemia and hypertension are also more than additive. The Nurse Health Study data indicate that the risk among former smokers decreases to the level of never smokers 10 to 14 years after the cessation of smoking. If a smoker stops smoking before the onset of disease she will experience a $24 \%$ reduction in the risk for mortality within 2 years of quitting smoking. This decline was apparent regardless of the quantity smoked or the duration of the habit. Clinical trials have studied smoking cessation as one component of compound intervention programs in which compliance varied widely and the estimates of risk reduction ranged from 18 to $65 \% .{ }^{17-19}$ The best health advice remains not to start smoking at all particularly at a younger age. These benefits are more substantial and occur sooner than in the case of delaying the cessation of smoking until the onset of disease.

\section{Hypertension}

Hypertention is one of the most common identifiable risk for CHD specially in women. More than 50\% were smoker in this study. Prospective observation studies have shown that high blood pressure is positively and independently associated with the risk of stroke, MI and mortality from all vascular causes in both men and women and in all ages. The marked benefit of pharmacologic therapy in malignant and severe hypertension have been reported in the primary prevention of stroke and MI. With drug therapy a reduction of mean diastolic blood pressure by $5 \mathrm{~mm} \mathrm{Hg}$ reduced mortality from all vascular causes by $21 \%$, the incidence of stroke by $42 \%$ and MI by $17 \%$ (reduction in stroke is much more remarkable than of MI). ${ }^{20-21}$ Although most studies have concentrated on elevated diastolic blood pressure, systolic blood pressure is a strong predictor of risk for CHD. Isolated systolic hypertension increases steadily after the age of 55 being more common in women than in men and it affects about $30 \%$ of these 65 to 75 years postmenopausal women. A reduction of $11 \mathrm{~mm} \mathrm{Hg}$ in systolic blood pressure resulted in an approximately $36 \%$ reduction in the incidence of stroke and $27 \%$ reduction in the combined end point of nonfatal infarction and coronary death. ${ }^{22,23}$

\section{Hyperlipidemia}

The elevated plasma levels of total cholesterol and low density lipoprotein (LDL) cholesterol are important risk factors for CHD. ${ }^{24,25}$ The relation between plasma cholesterol and coronary events appears to be stronger if levels are at elevated rather than avearge value. Clinical trails have shown that lowering elevated level of cholesterol and LDL cholesterol levels prevent both first and recurrent coronary events. A $10 \%$ reduction in cholesterol level was associated with a $10 \%$ reduction in the risk of CHD in less than 4 years of treatment and $20 \%$ reduction with longer treatment period. ${ }^{26,27}$ On average $10 \%$ reduction in the cholesterol level are achieved with dietary therapy and 20\% reduction with drug therapy. Dietary, environmental and genetic factors all have important roles in determining blood levels of cholesterol as well as rates of coronary disease. ${ }^{25-27}$ Levels of high density lipoproteins (HDL) cholesterol in contrast to those of LDL and total cholesterol are inversely related to the risk of CHD. In observational data from multiple risk factor interventional and lipid research clinic prevalence study ${ }^{28}$ this inverse relation between HDL cholesterol and CHD remained significant even after control for age, level of LDL cholesterol, triglyceride level, body mass index, blood pressure and smoking status. Changes in the physicians practices and in the level of awareness among patients suggest that continued cholesterol intervention will have a substantial effect in further reducing the rates of acute myocardial as well as death from coronary disease in postmenopausal women as well as in men.

\section{Diabetes Mellitus}

Diabetes mellitus (DM) accelerates the atherogenesis and increase the risk of MI particularly in women. In populationbased studies, the age-adjusted mortality rate from CHD is 3 to 7 times higher among diabetes women than among people without diabetes. ${ }^{29}$ Obesity and family history of diabetes are major determinants of development of noninsulin-dependent diabete smellitus (NIDDM). Coronary risk factors, such as hypertension, dyslipoproteinemia as well as clinically manifest cardiovascular disease, are present in excess at the time of diagnosis of NIDDM. Diabetes is a far greater risk factor for women than men, women 45 years are twice as likely as men to develop 
diabetes. ${ }^{30,31}$ Diabetes has an adverse effect on the in-hospital and long-term prognosis after MI much more worse in women. More women than men who undergo myocardial revascularization procedure are diabetic which probably contributes to less favorable outcome in women. These interrelations suggest the presence of coexisting genetic or metabolic factors or both in the casual pathway common to all these conditions. ${ }^{32}$ Mechanisms postulated for its independent effect are an increased tendency to thrombosis, cardiac autonomic neuropathy and diabetic cardiopathy. The deleterious consequences of diabetes increase markedly with duration and in the presence of other coronary risk factors. Recommendation for the prevention of heart disease in diabetic persons include the favorable modification of other coronary risk factors. Of paramount importance, however, is the primary prevention of NIDDM by the avoiding or treatment of obesity. Recent studies also suggest a promising role for physical activity in the prevention of NIDDM.

\section{Obesity}

Obesity is a well-established cause of diabetes mellitus, hypertension and lipid abnormalities and atherosclerosis. Nearly a doubling of risk was observed among the obese as compared with subjects at desirable weight. Recently in a study in women the age and smoking adjusted relative risk of coronary disease was 3.3 (95\% confidence interval: 2.3-4.5) among severely obese women (body mass index $>29$ ) and even mild to moderate overweight (body mass index: 25-28) was associated with an $80 \%$ increase in risk. ${ }^{32}$ The cardiovascular risk of obesity were amplified by other coronary risk factors. Several studies have also suggested that the distribution of fat particularly fat deposition in the abdomen and upper body may have important effects on the risk of myocardial infarction. ${ }^{32,33}$ The effect of weight reduction on the risk of coronary heart disease remains uncertain because of small number of subjects with sustained weight loss in prospective studies. ${ }^{3}$ Weight reduction has favorable effects on several coronary risk factors including glucose tolerance, blood pressure and serum lipid levels. Weight reduction induced by either dietary or increased exercise produce comparable beneficial changes in heart disease and triglyceride level. ${ }^{32}$ Helping patients achieve and maintain weight loss however is one of the most vexing problem in clinical practice. Effective programs of treatment must be multifaceted including a hypocaloric diet, nutrition education, behavior modification, counceling, emphasis on increased physical activity and physiological and social support. ${ }^{3}$ The estimated reduction in the risk of MI associated with maintaining an ideal body weight as compared with being obese ( $>20 \%$ above desirable weight) is 30 to $35 \%$. $^{32-34}$

\section{Physical Activity}

Physical exercises improve functional work capacity and usually lowers heart rate and blood pressure, two major determinants of myocardial oxygen demand. In addition exercises help to reduce weight, lower platelet adhesiveness, increase the HDL level, inhances fibrinolysis and lessen the adrenergic response to stress. It is biologically plausible that a lack of physical exercises independent of other coronary risk factors would be a major risk for CHD.

There is a large body of evidence in support of an association of physical activity and CHD. Most of the evidence for an association of habitual physical activity with CHD was largely established in studies among men only. In case control studies habitual light physical activity and regular physical activity were associated with a 50\% decreased risk of myocardial infarction and sudden death in women. ${ }^{34}$ In a cohort study of Swedish women leisure time physical activity was not significantly associated with the risk of MI after adjustment for other coronary risk factors. ${ }^{35}$ In a cohort study of Finnish women, ${ }^{36}$ physical activity at work but not leisure time physical activity was significantly associated with a decreased risk of MI. A strong decreased risk of MI was associated with walking for exercise. In addition leisure time physical activity was associated with other health behaviors such as eating low fat diet, control on smoking and alcohol consumption. A $50 \%$ decrease risk of MI among postmenopausal women was seen who participated in modest level of non strenuous physical activities. It was found that the near maximal benefit with total energy expenditure corresponding to 30 to 45 minutes of walking for exercise three times a week. ${ }^{37}$ Similar recommendation was given by the American Heart Association for health promotion that 30 to 60 minutes sessions of dynamic exercise of the large muscles 3 to 4 times a week. Physicians should recommend participation in nonstreneous activities, such as walking to women as well as men.

\section{Alcohol Consumption}

Although heavy alcohol use has been shown to increase the risk of MI and stroke and mortality from CHD, there is a substantial body of observational epidemiologic evidence to suggest that moderate consumption of alcohol reduces the risk of heart disease. ${ }^{3}$ A large prospective cohort study has shown inverse association between moderate alcohol consumption and the risk of MI. The Framingham study ${ }^{37}$ 
observed 30\% reduction in risk among men and women who consumed more than $30 \mathrm{gm}$ of alcohol per month. The Nurses Health Study ${ }^{6}$ observed a $40 \%$ reduction in risk among women who consume 10 to $15 \mathrm{gm}$ of alcohol per day compared with nondrinkers. The reduction in the risk of MI appears to be independent of the type of beverage consumed suggesting that alcohol itself rather than other components of the drinks is responsible for the observed effect. The protective effect of alcohol on CHD include alcohol-mediated increase in HDL cholesterol including its subfractions HDL2 or HDL3 and both subfractions are inversely related to risk of MI. ${ }^{38}$ Other postulated mechanism include the effects of alcohol on platelets aggregation, the release of plasminogen activator or fibrinogen. ${ }^{38}$ However heavy consumption of alcohol has been implicated in accidents, cirrhosis, cancer and other adverse outcomes. The difference between drinking small to moderate quantities of alcohol and drinking large amounts may mean the difference between preventing and causing the disease. Any clinical recommendation based on epidemiological evidence should therefore be cautions. No data from randomized trails are available.

\section{Prophylactic Aspirin}

Primary prevention: Aspirin is an irreversible inhibitor of platelet cyclooxygenase activity and thereby interferes with platelet activation. Chronic administration of low dose aspirin of 75 to $325 \mathrm{mg}$ orally per day has been shown to reduce the coronary events in asymptomatic men and women. Six large scale trials have assessed the benefit of low dose aspirin in the prevention of cardiovascular disease. These studies suggested a benefit of prophylactic aspirin in primary prevention of MI and ischemic stroke. ${ }^{38}$ The Women's Health study (WHS) ${ }^{39}$ addressed the benefit to risk ratio of aspirin therapy for primary prevention of $\mathrm{CHD}$ specifically in women. In this study a $100 \mathrm{mg}$ dose of aspirin every other day lowered the risk of stroke but did not affect the risk of MI or death from other cardiovascular causes in this group of initially healthy women who were 45 or older. ${ }^{39}$ However, among the study participants 65 or older the $100 \mathrm{mg}$ alternate day aspirin dose reduced the risk of major CHD events by $26 \%$. The WHS also looked at primary prevention using aspirin dose below $75 \mathrm{mg}$ which produced a suboptimal affect. ${ }^{40}$ In 2004, AHA offered more specific guidelines for women recommending aspirin for women whose 10 years risk of a first coronary events exceed $20 \%$ and a consideration for using it in women whose 10 years risk is between 10 and $20 \%{ }^{41}$
Secondary prevention: Various secondary preventive measures are at least partly responsible for the improvement in the long term mortality and morbidity rates after acute MI. Long-term treatment with an antiplatelet agent usually aspirin after MI is associated with a $25 \%$ reduction in the risk of recurrent MI, stroke or cardiovascular mortality (36 fewer events for every 1,000 patients treated). In addition in patients taking aspirin chronically MI tend to be smaller and are more likely to be non-Q-wave in nature. ${ }^{42}$ Administration of this drugs should be considered in all patients with CHD in the absence of gastrointestinal bleeding, allergy or dyspepsia. Clopidogrel (300 mg loading and $75 \mathrm{mg}$ /day) is an oral agent that blocks ADP receptormediated platelet aggregation. Clopidogrel with aspirin can improve coronary outcomes when given to patients for 1 year after an episode of unstable angina but with some increase in the risk of bleeding. In the CURE trial a combination of clopidogrel with aspirin confer a $20 \%$ relative reduction in cardiovascular death, MI or stroke compared with aspirin alone in both low and high-risk patient with CHD but to be associated with a moderate (absolute 1\%) increase in serious bleeding. ${ }^{43}$

\section{CONCLUSION}

The magnitude of $\mathrm{CHD} / \mathrm{MI}$ is higher in women especially in those who are hypertensive, diabetes, low endogenous hormone level, lower body weight, and high fat proportion. It is paramount important to bring increase awareness of CHD in women because almost $65 \%$ of deaths occur in those with no previous symptoms. With the aging of population more women than men now die of coronary heart disease each year unless women take preventive measures throughout their life.

\section{REFERENCES}

1. Marynard C, Litwin PE, Martin JS, Weaver ND. Gender difference in the treatment and outcome of acute myocardial infarction: results from myocardial infarction Triage and intervention Registry. Arch Intern Med 1992;1542:972-976.

2. Rajadurai J, Lpez EA, Rahajoe AV, et al. Womens cardiovascular health: prospectives from South East Asia. Nat Rev Cardol 2012;24(9):464-477.

3. Stangl V, Witzel V, Baumann G, et al. Current diagnostic concepts to detect Coronary artery disease in women. European Heart Journal 2008;29:707-717.

4. Tofler GH, Stone PH, Muller JE, Braunwald E. Mortality for women after acute myocardial infarction: Milis study group. Am J Cardid 1989;64:256.

5. Bell MB, Holmes DR Jr, Borger PB, et al. The changing in hospital mortality of women undergoing percutaneous tranluminal coronary angioplasty. JAMA 1993;269:2091-2095. 
6. Navette K, Wenger R. Coronary heart disease: an older women's major health risk. BMJ 1998;13:124-152.

7. Weintraub WS, Wenger NK, Jones EL, Craner JM, Guyton RA. Changing clinical characteristics of coronary surgery patients. Difference between men and women. Circulation 1993;88: 79-86.

8. Cooper R, Cutlen J, Desvigne-Nicken P, et al. Trends and disparities in coronary heart disease, stroke and other cardiovascular disease in the United States: findings of the national conference on cardiovascular disease prevention. Circulation 2000;102:3137.

9. Manson JE, Ridker PM, Sulterfield S, Herbort P, et al. The primary prevention of myocardial infarction. N Eng J Med 1992;326:1406-1416.

10. Campbell NC, Thain J, Deans HG, Lewis D, et al. Secondary prevention in coronary heart disease: baseline survey of provision in general practice. BMJ 1998;316:1430-1434.

11. Bowker TJ, Clayton TC, Ingham J, et al. A Bristish Cardiac Society Survey of the potential for the secondary prevention of coronary disease: ASPIRE (Action on secondary prevention through intervention to reduce events). Heart 1996;75:334-342.

12. Hulley S, Furberg C, Barrett-Connor E, et al. Noncardiovascular disease outcome during 6 to 8 years of hormone therapy. Heart and Estrogen/Progestin replacement study follow-up (HERSII) JAMA 2002;288:58.

13. Rossouw JE, Prentice RL, Manson JE, et al. Postmenopausal hormone therapy and risk of cardiovascular disease by age and years since menopause. JAMA 2007;297:1465.

14. Rossouw JE, Anderson GL, Prentice RL, et al. Risk and benefit of estrogen plus progestin in healthy postmenopausal women. JAMA 2002;288:321-333.

15. Department of Health and Human Services. Reducing the health consequences of smoking. 25 years of progress: a report of the surgeon general. Washington DC. Government Printing Office DHHS Publication No. (CDC) $8411 ; 1989$.

16. Mosca L, Collins P, Herrington DM, et al. Hormone replacement therapy and cardiovascular disease: a start for healthcare professionals from the American heart association. Circulation 2001;104:499.

17. Willett WC, Green A, Stamfer MJ, et al. Relative and absolute excess risk of CHD among women who smoke cigarettes. N Eng J Med 1987;314:103-109

18. Kawachi I, Coldtiz GA, Stamfer MJ, et al. Smoking cessation in relation to total mortality rates in women. Ann Intern Med 1993;119:992-1000.

19. Ockene JK, Kuller LH, Svendsen KH, Meilah E. The relationship of smoking cessation to coronary heart disease and lung cancer in the multiple risk factor interventional trail (MRFIT). Am J Public Health 1990;80:954-958.

20. MacMohan S, Peto R, Cusler J, et al. Blood pressure, stroke and coronary heart disease part I. Prolong differences in blood pressure: prospective observational studies corrected for the regression dilution bias. Lancet 1990;335:765-774.

21. Collins R, Peto R, MacmMohan S, et al. Blood pressure, stroke and coronary heart disease part 2 short term reduction in blood pressure: overview of randomized drugs trial in their epidermiologic context. Lancet 1990;335:827-838.

22. Kannel WB, Dawber TR, Megee DL. Perspective on systolic hypertension: The framingham Study. Circulation 1980;61: 1179-1182.
23. Prevention of strokes by antihypertensive drug treatment in older persons with isolated systolic hypertension in the elderly program (SHEP). SHEP Co-operative Research Group. JAMA 1991;265:3255-3264.

24. Kannel WB. Range of serum cholesterol values in the population developing coronary artery disease. Am J Cardiol 1995;76: 69-77.

25. Sacks FM, Pfeffer MC, Moye LA, et al. The effect of pravastatin on coronary events after myocardial infarction in patients with average cholesterol level. N Eng J Med 1996;335:1001-1008.

26. Pharoah PD, Hollingworth W. Cost-effectiveness of lowering cholesterol concentration with statins in patients with or without preexisting coronary heart disease life table method applied to health authority population. BMJ 1996;312:1643-1648.

27. Miller NE, Forde OH, Thelle DS, Mjos OD. The Tromsq Heart study; high density lipoproteins and coronary heart disease: a prospective case-control study. Lancet 1977;1:965-968.

28. Jacobs DR Jr, Mebane IL, Bangdiwada SI, Onqui MH, Tyroer HA. High density lipoprotein cholesterol as a predictor of cardiovascular disease mortality in men and women. The followup study of lipid research clinics prevalence study. Am J Epidemiol 1990;131:32-47.

29. Kannel WB, Mcgee DL. Diabetes and cardiovascular disease. The Framingham study. JAMA 1979;241:2035-2038.

30. Kanaya AM, Herrington D, Vittinghoff E, et al. Glycemic effects of postmenopausal hormone therapy: The heart and estrogen/ progestin replacement. A randomized, Double-blind placebocontrolled trial. Ann Intern Med 2003;138:1-9.

31. Margolis KL, Bonds DE, Rodabough RJ, et al. Effects of oestrogen and progestin on the incidence of diabetes in post menopause women: results from the women's health initiative hormone Trial Diabetologia 2004;47:1175.

32. Manson E, Colditz GA, Stanff MJ, et al. A prospective study of obesity and risk of coronary heart disease in women. N Eng JM Ed 1990;322:882-889.

33. Hubert HB, Feinleib M, McNamora PM, Castelli WP. Obesity as an independent risk factor for cardiovascular disease. A 26 years follow-up of participants in the Framingham heart study. Circulation 1983;67:968-977.

34. Lemaitre RN, Heckbest SR, Psaty BM, Siscovick DS. Leisure time physical activity and the risk of nonfatal myocardial infarction in postmenopausal women. Arch Intern Med 1995;27:2302-2308.

35. Lapidus L, Benglsson C. Socioeconomic factors and physical activity in relation to cardiovascular disease and death: a 12 years follow-up of participants in a population study of women in Gothenburg. Sweden Br Heart Jr 1985;55:295-301.

36. Kushi LH, Fee RM, Folson AR, NINK P, et al. Physical activity and mortality in postmenopausal women. JAMA 1997;277: 1287-1292.

37. Kannel WB, Gorden T. Some characteristics of the incidence of cardiovascular disease and death: framingham study: 16 years follow-up. Section 26 of the framingharm study. Washington DC: Government Printing Office; 1970.

38. Stampfer MJ, Cololitz GA, Willett WD, Speizar FF, Hennenken $\mathrm{CH}$. The prospective study of moderate alcohol consumption and risk of coronary disease and stroke in women. N Eng J Med 1988;319:267-273

39. Berger JS, Roncaglioni MC, Avanzini F, et al. Aspirin for the primary prevention of cardiovascular events in women and men. 
A sex-specific meta-analysis of randomized control trials. JAMA 2006;295:3061.

40. Ridker PM, Cook NR, Lee IM, et al. A randomized trial of low dose aspirin in the primary prevention of cardiovascular disease in women. N Eng J Med 2005;352:1293.

41. Pearson TA, Blair SN, Daniel SR, et al. AHA guidelines for primary prevention of cardiovascular disease or stroke: 2002 update: Circulation 2002;106:388.

42. Libby P, Bonow RO, Mann DL, Zipes DP, Braunwal E. Braunwald's heart disease: cardiac protection with aspirin. Elsevier Saunder Publisher. 8th ed. 2008. 1133 p.

43. Antithrombotic Trialists' Collaboration. Collaborative metaanalysis of randomised trial of antiplatelet therapy for prevention of death, MI and stroke in high-risk patients. BMJ 2002;324:71.

\section{ABOUT THE AUTHORS}

\section{P Pradhan (Corresponding Author)}

Professor and Head, Department of Obstetrics and Gynecology Nepal Medical College, Jorpati, Kathmandu, Post Box 13344, Nepal e-mail: drpramilapradhan@gmail.com

\section{AV Upadhya}

Senior Physician Cardiologist, Medicure Hospital; Ex-Senior Physician and Cardiologist, Bir Hospital, Kathmandu, Nepal 